\begin{tabular}{|c|l|}
\hline Title & Time of-flight observation of electron swarm in methane \\
\hline Author(s) & Hasegawa, H.; Date, H.; Y oshida, K.; Shimozuma, M. \\
\hline Citation & $\begin{array}{l}\text { Journal of A pplied Physics, 105(11), 113308 } \\
\text { https://doi.org/10.1063/_.3142322 }\end{array}$ \\
\hline Issue Date & 2009-06 \\
\hline Doc URL & http://hdl.handle.net/2115/38722 \\
\hline Rights & $\begin{array}{l}\text { Copyright 2009 A merican Institute of Physics. This article may be downloaded for personal use only. Any other use } \\
\text { requires prior permission of the author and the A merican Institute of Physics. }\end{array}$ \\
\hline Type & article \\
\hline File Information & JAPIA U10511113308_1.pdf \\
\hline
\end{tabular}

Instructions for use 


\title{
Time-of-flight observation of electron swarm in methane
}

\author{
H. Hasegawa, ${ }^{1}$ H. Date, ${ }^{2, a)}$ K. Yoshida ${ }^{3}$ and M. Shimozuma ${ }^{4}$ \\ ${ }^{1}$ Tomakomai National College of Technology, Tomakomai 059-1275, Japan \\ ${ }^{2}$ Faculty of Health Sciences, Hokkaido University, Sapporo 060-0812, Japan \\ ${ }^{3}$ Kitami Institute of Technology, 090-8507, Japan \\ ${ }^{4}$ Hokkaido Institute of Technology, Sapporo 006-8585, Japan
}

(Received 26 February 2009; accepted 2 May 2009; published online 10 June 2009)

\begin{abstract}
This paper reports on the evolution of an isolated electron swarm, which is experimentally observed as spatial distributions at every moment. This observation is assumed to directly correspond to the conventional time-of-flight theory. We have measured the spatial distribution of electrons using a double-shutter technique in the drift tube, where a shutter electrode to collect electrons can be slid along the field $(E / N)$ direction in order to capture a relative electron number at a certain range of location. As a typical parameter defined by this spatial distribution, the center-of-mass drift velocity $\left(W_{r}\right)$ is determined for methane gas. The result is compared with the mean-arrival-time drift velocity $\left(W_{m}\right)$ defined from the arriving electron number at fixed positions. We have also performed a theoretical analysis in which a Fourier transformed Boltzmann equation is solved to deduce both of the drift velocities from a dispersion relationship. The difference between $W_{r}$ and $W_{m}$ at high $E / N \mathrm{~s}$ (above $200 \mathrm{Td}$ ) is clearly ascertained in the experimental and theoretical investigations, which is attributable to the occurrence of ionization events. (C) 2009 American Institute of Physics.
\end{abstract}

[DOI: 10.1063/1.3142322]

\section{INTRODUCTION}

The term electron swarm is used for a state of the electron ensemble in weakly ionized gases, which often exhibits the nature of equilibrium forming a macroscopic steadystate, i.e., the hydrodynamic equilibrium state. The macroscopic behavior of an isolated electron swarm can be described by the Boltzmann equation, leading to the continuity equation by integrating over velocity space. The swarm parameters, such as the drift velocity and the diffusion coefficient appearing in the continuity equation, have an inclination to converge to a set of constant values as time elapses (e.g., Kumar et al. ${ }^{1}$ ). In this regard, it may be appropriate to refer to the electron ensemble in this kind of equilibrium state as an "electron swarm."

The spatiotemporal evolution of the electron swarm has been studied since 1969, e.g., by Parker and Lowke, ${ }^{2}$ Thomas, ${ }^{3}$ Skullerud, ${ }^{4}$ Tagashira et al., ${ }^{5}$ Kumar et al., ${ }^{1}$ and other investigators. These investigations describe the electron swarm envisaging the spatial distribution at a certain time, which accords to the continuity equation (or "diffusion equation") derived from the Boltzmann equation. We call this approach to capture the swarm behavior the time-offlight (TOF) method hereafter. Contrary to this, Kondo and Tagashira ${ }^{6}$ presented the arrival-time-spectra (ATS) method in which the swarm is observed at a certain location in the drift tube and the arrival-time distribution of electrons is treated to deduce macroscopic parameters emerging in another type of continuity equation. This continuity equation was obtained by interchanging time and space variables in the conventional continuity equation (see also Date $e t a .^{7}$ ),

\footnotetext{
a) Author to whom correspondence should be addressed. Present address: N12-W5, Kita-ku, Sapporo 060-0812, Japan. Electronic mail: date@hs.hokudai.ac.jp. Tel.: +81-11-706-3423. FAX: +81-11-706-4916.
}

where the spatial derivative of electron number is expanded in a series of time derivatives with higher orders.

The authors have measured the arrival-time distribution of electrons by a double-shutter electrode technique based on the ATS method, ${ }^{8,9}$ demonstrating the ATS method experimentally in order to achieve a direct comparison of the parameters between the theoretical and experimental. In the course of the ATS measurement, we found that our apparatus enables us to measure also the spatial distribution of an isolated swarm under moderate field conditions (see next section) by moving the double-shutter electrode to collect incoming electrons along the drift direction (electric field direction) and to deduce directly a typical parameter, the drift velocity.

The measurement of the swarm using the variable length drift tube has been reported by several authors. Schmidt ${ }^{10}$ applied the drift tube to study the electron swarm under the influence of electric and magnetic fields, and Nakamura ${ }^{11}$ determined the electron swarm parameters in some gases using the same type of apparatus as ours. Hegerberg et al. ${ }^{12}$ measured the ion mobility using the variable length drift tube. Fletcher ${ }^{13}$ performed the direct measurements provided by the photon flux technique with the drift tube. However, the investigations on the electron swarm with the variable length drift tube so far are directed to the electron behavior under low $E / N$ electric fields below $100 \mathrm{Td}$ in which the ionization events hardly occur. The ionization process is, needless to say, essential in the gas discharges and the plasma reactors. It has been asserted, resorting to the simulation, that in the condition with ionization process, the macroscopic swarm parameters, such as the drift velocity and the diffusion coefficient, of an isolated electron swarm are affected quantitatively depending on their definitions. ${ }^{5-7,14-17} \mathrm{~A}$ typical example is rendered by that the mean drift velocity of electrons in an isolated swarm is different from the center- 
of-mass drift velocity of electrons "in general" when ionization and/or attachment processes occur. ${ }^{5}$ The knowledge of this effect may be of great importance in treating the behavior of charged particles with production and annihilation processes by external electric and/or magnetic fields.

In this paper, the drift velocities determined experimentally with two types of observational principle are presented and compared with those by a theoretical analysis. The double-shutter electrode technique with the variable length drift tube is applied to make the TOF observation of electron swarms in methane gas. In the theoretical analysis, the drift velocities are deduced by using a Fourier transformed Boltzmann equation ${ }^{14}$ to compare with the experimental results. To our knowledge, the experimental observation presented here is the first demonstration to directly observe the TOF evolution of the electron swarm in the ionization regime, which corresponds to the TOF theory associated with the effect of nonconservative condition of electron number on the swarm parameters.

\section{EXPERIMENTAL METHOD}

\section{A. Double-shutter electrode in drift tube}

The experimental apparatus used in the present experiment is the same as that for the ATS method described by Hasegawa et al., ${ }^{8}$ but is utilized differently, where the double-shutter is moved little by little along the field direction in the drift chamber for capturing the spatial distribution of electron swarms. Figure 1 shows a schematic diagram of the chamber and its internal configurations. The initial electrons are generated at photocathode (PC) and released by shutter-1 $\left(\mathrm{S}_{1}\right)$ periodically as a starting electron pulse. A combination of shutter-2 $\left(\mathrm{S}_{2}\right)$ and collector electrode $(\mathrm{C})$ to collect the coming electrons is linearly movable from 0 to $100 \mathrm{~mm}$ in the longitudinal direction. Shutter-2 can cut out the coming electrons of the swarm at fixed locations after a certain delay from the timing of shutter-1 open, which is repeatedly performed to collect a sufficient quantity of electron charge.

\section{B. Experimental condition}

We have measured the collected charge at locations from 5 to $60 \mathrm{~mm}$ in $1 \mathrm{~mm}$ increments along the drift space under the constant reduced fields of $E / N=200-500 \mathrm{Td}(1 \mathrm{Td}$ $\left.=10^{-17} \mathrm{~V} \mathrm{~cm}^{2}\right)$. Although the set of shutter-2 $\left(\mathrm{S}_{2}\right)$ and collector electrode $(\mathrm{C})$ is movable up to $100 \mathrm{~mm}$, the maximum length was limited to around $60 \mathrm{~mm}$ in the present experiment because of the voltage limit applied to between $S_{1}$ and $\mathrm{S}_{2}$ (the applied voltage should be higher as the drift length becomes longer to maintain constant $E / N \mathrm{~s}$, but the breakdown may occur in the chamber if the voltage is too high). On the other hand, the minimum distance was set above 5 $\mathrm{mm}$ to keep the field uniform between the shutters.

The experimental conditions are summarized in Table I. Frequency of the pulse onto the double-shutter is $500 \mathrm{kHz}$, and the delay time between $\mathrm{S}_{1}$ and $\mathrm{S}_{2}$ is 1-3 ns. Purity of methane used here is $99.999 \%$.

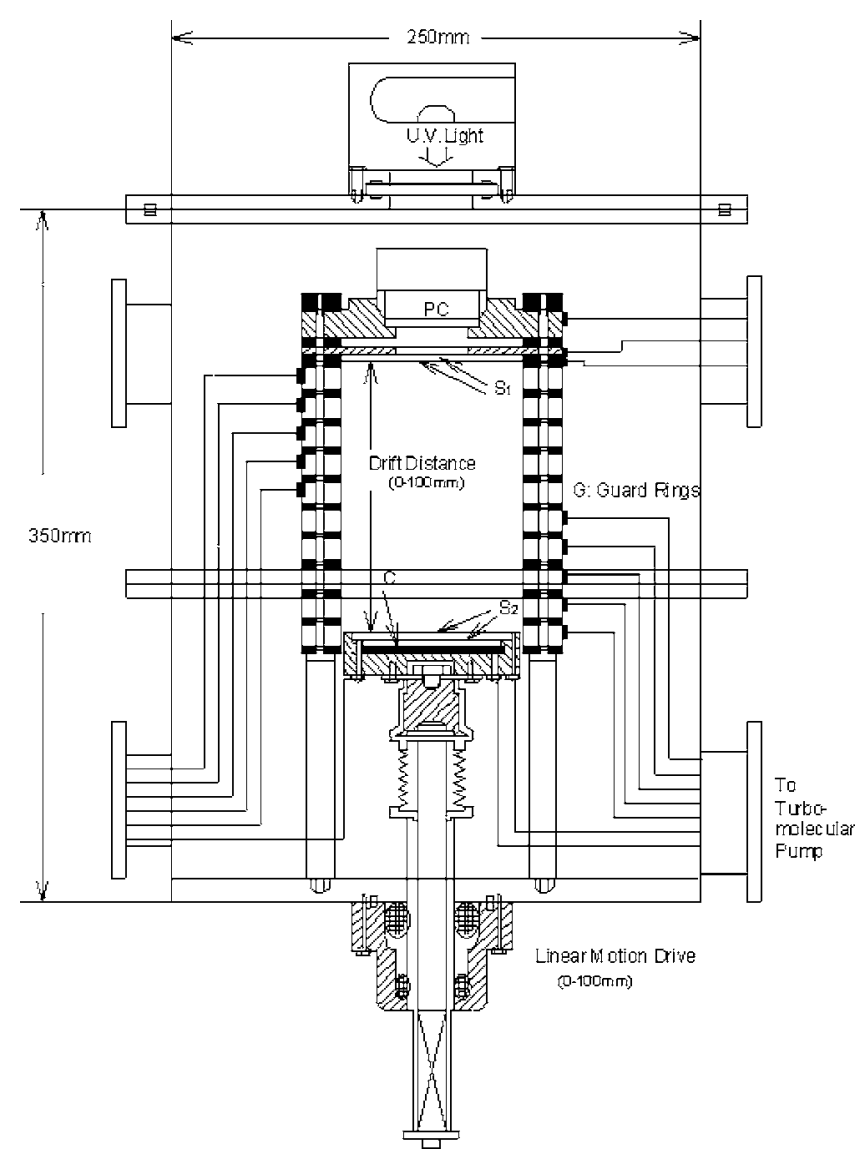

FIG. 1. A schematic diagram of apparatus used in the experiment. The symbols represent as follows: $\mathrm{PC}$, photo-cathode; $\mathrm{S}_{1}$ and $\mathrm{S}_{2}$, first and second shutters; $\mathrm{C}$, collector; and $\mathrm{G}$, guard rings.

\section{COMPUTATIONAL METHOD}

Corresponding to the experimental TOF observation, we have performed theoretical analysis of the Boltzmann equation for methane gas. In the present analysis, a Fourier transformed Boltzmann equation method ${ }^{14}$ was used to calculate the swarm parameters. The method enables us to determine a set of the TOF and ATS parameters at once. The methodology is presented briefly as follows.

Starting from the Boltzmann equation for electrons as

$$
\left(\frac{\partial}{\partial t}+\vec{v} \cdot \frac{\partial}{\partial \vec{r}}+\frac{e \vec{E}}{m} \cdot \frac{\partial}{\partial \vec{v}}+J\right) f(\vec{r}, \vec{v}, t)=0,
$$

where $\vec{v}=\left(v_{x}, v_{y}, v_{z}\right), \vec{r}=(x, y, z)$, and $J$ represents the collision operator, the equation in terms of $z$-direction is obtained by integrating Eq. (1) over $d x d y$ as

$$
\left(\frac{\partial}{\partial t}+v_{z} \frac{\partial}{\partial z}+\frac{e E}{m} \frac{\partial}{\partial v_{z}}+J\right) f(z, \vec{v}, t)=0 .
$$

Here, $f(z, \vec{v}, t)=\iint f(\vec{r}, \vec{v}, t) d x d y$ and the electric field $E$ is parallel to $z$-direction. If we assume the solution $f(z, \vec{v}, t)$ to be in a form of Fourier integral ${ }^{2,5}$ as

$$
f(z, \vec{v}, t)=\frac{1}{2 \pi} \int \hat{f}(k, \vec{v}) e^{i k z-\omega t} d k,
$$

Eq. (2) can be transformed into 
TABLE I. Conditions of the experiment.

\begin{tabular}{lccccccc}
\hline \hline & \multicolumn{7}{c}{ Field strength $E / N(\mathrm{Td})$} \\
\cline { 2 - 8 } & 200 & 250 & 300 & 350 & 400 & 450 & 500 \\
\hline Temperature $\left({ }^{\circ} \mathrm{C}\right)$ & 17.5 & 17.4 & 18.7 & 17.6 & 18.5 & 17.5 & 18.2 \\
Gas pressure (Torr) & 1.0 & 0.6 & 1.0 & 0.4 & 0.5 & 0.3 & 0.3 \\
Distance between $\mathrm{S}_{1}$ and $\mathrm{S}_{2}(\mathrm{~mm})$ & $10-40$ & $5-50$ & $10-30$ & $5-60$ & $5-50$ & $5-60$ & $5-50$ \\
\hline
\end{tabular}

$$
\left(i k v_{z}+\frac{e E}{m} \frac{\partial}{\partial v_{z}}+J\right) \hat{f}(k, \vec{v})=\omega \hat{f}(k, \vec{v}) .
$$

Here, Eq. (3) is assumed to be integrable under the condition that the electron swarm is in the hydrodynamic equilibrium state at a time after a long enough (but not infinite) period from the initial moment of the swarm generation. It is noted that Eq. (4) exhibits a form of the eigenvalue problem in which the solution affords us the relationship between $\omega$ and $k$, namely, "dispersion relation." The relation described later is known to correspond to the lowest eigenvalue, which is given in the hydrodynamic regime. ${ }^{15,18}$

Besides the transformation above, the solution $f(z, \vec{v}, t)$ for Eq. (2) can be expressed in a multiterm formula by the density gradient expansion method (e.g., Kumar et al. ${ }^{1}$ ) as

$$
f(z, \vec{v}, t)=\sum_{K} f^{(K)}(\vec{v}) \cdot\left(-\frac{\partial}{\partial z}\right)^{K} n(z, t),
$$

and the continuity equation of electron density is also given by

$$
\frac{\partial n}{\partial t}=\sum_{K} \omega^{(K)}(\vec{v}) \cdot\left(-\frac{\partial}{\partial z}\right)^{K} n(z, t) .
$$

Thus, Eqs. (5) and (6) are transformed into

$$
\hat{f}(k, \vec{v})=\sum_{K} f^{(K)}(\vec{v})(-i k)^{K}
$$

and

$$
\omega=-\sum_{K} \omega^{(K)}(-i k)^{K},
$$

respectively. Here, $n(z, t)=\int f(z, \vec{v}, t) d \vec{v}=(1 / 2 \pi) \int \hat{f}(k) e^{i k z-\omega t}$ $\times d k$. If we put $p \equiv i k$, then Eq. (8) comes to $\omega(p)=$ $-\Sigma_{K} \omega^{(K)}(-p)^{K}$, and the coefficients in the right hand side, $\omega^{(K)}(K=0,1,2 \ldots)$, are found to be corresponding to the TOF parameters as

$$
\begin{aligned}
-\omega(p) & =\omega^{(0)}-\omega^{(1)} p+\omega^{(2)} p^{2}-\omega^{(3)} p^{3}+-\ldots \\
& =R_{i}-W_{r} p+D_{L} p^{2}-D_{3} p^{3}+-\ldots
\end{aligned}
$$

Here, $R_{i}$ is the effective ionization frequency, $W_{r}$ is the center-of-mass drift velocity, $D_{L}$ is the longitudinal diffusion coefficient, and $D_{K}(K=3,4, \ldots)$ are the higher coefficients. ${ }^{5}$ Contrary to this, if we obtain the inverse of Eq. (9) as

$$
p=\sum_{K} \alpha^{(K)}(-\omega)^{K} \quad(K=0,1,2,3 \ldots)
$$

the coefficients $\alpha^{(K)}$ represent ATS parameters, ${ }^{6}$ i.e.,

$$
\begin{aligned}
p(\omega) & =\alpha^{(0)}-\alpha^{(1)} \omega+\alpha^{(2)} \omega^{2}-\alpha^{(3)} \omega^{3}+-\ldots \\
& =\alpha_{T}-\frac{1}{W_{m}} \omega+\alpha^{(2)} \omega^{2}-\alpha^{(3)} \omega^{3}+-\ldots,
\end{aligned}
$$

where $\alpha_{T}$ is the Townsend effective ionization coefficient and $W_{m}$ is the mean-arrival-time drift velocity. Consequently, we can see that once the relation between $\omega$ and $i k$ (or $p$ ) is obtained from the Eq. (4), the TOF and ATS parameters can be determined by curve fitting to this relation with polynomial equations [Eqs. (9) and (11)].

In order to solve Eq. (4), we used the Legendre expansion technique, where $\hat{f}(k, \vec{v})$ is expanded as $\hat{f}(k, \vec{v})$ $=\sum_{\ell} \hat{f}_{\ell}(k, v) P_{\ell}\left(\cos \theta_{v}\right)$. Here, $\theta_{v}$ is the angle (zenith angle) between $z$-axis and the electron velocity. Taking account of the formulae on Legendre polynomial, $\cos \theta_{v}=\left[(\ell+1) P_{\ell+1}+\ell P_{\ell-1}\right] /\left[(2 \ell+1) P_{\ell}\right], \quad \partial / \partial v_{z}=\cos \theta_{v}$ $\times(\partial / \partial v)-\left(\sin \theta_{v} / v\right)\left(\partial / \partial \theta_{v}\right)$, and $\sin \theta_{v}\left(\partial P_{\ell} / \partial \theta_{v}\right)=[\ell(\ell+1)$ $\left.\times\left\{P_{\ell+1}-P_{\ell-1}\right\}\right] /(2 \ell+1)$, we obtain

$$
\begin{aligned}
\sum_{\ell}[ & -\omega(p) P_{\ell}+p v \frac{(\ell+1) P_{\ell+1}+\ell P_{\ell-1}}{2 \ell+1} \\
& +\frac{e E}{m} \frac{(\ell+1) P_{\ell+1}+\ell P_{\ell-1}}{2 \ell+1} \frac{\partial}{\partial v} \\
& \left.-\frac{e E}{m} \frac{1}{v} \frac{\ell(\ell+1)}{2 \ell+1}\left(P_{\ell+1}-P_{\ell-1}\right)+J_{\ell} P_{\ell}\right] \hat{f}_{\ell}(p, v)=0 .
\end{aligned}
$$

The relationship between $\omega$ and $p$ is given by integrating Eq. (12) over $d v$ as

$$
\omega(p)=\frac{\frac{p}{3} \int v^{3} \hat{f}_{1}(p, v) d v-\int N \bar{q}_{i} v^{3} \hat{f}_{0}(p, v) d v}{\int v^{2} \hat{f}_{0}(p, v) d v},
$$

where $N$ is the number density of gas molecules and $\bar{q}_{i}$ represents the ionization cross section minus attachment cross section. Note that the relation of Eq. (13) is actually determined for the variable $p$ at discrete points by a relaxation method to solve the following simultaneous equations: 



FIG. 2. Collector current at second shutter $\left(\mathrm{S}_{2}\right)$ as a function of drift distance: (a) $E / N=200 \mathrm{Td}$, (b) $E / N=300 \mathrm{Td}$, (c) $E / N=400 \mathrm{Td}$, and (d) $E / N$ $=500 \mathrm{Td}$.

$$
\begin{aligned}
-\omega(p) \hat{f}_{\ell}+p v\left(\frac{\ell}{2 \ell-1} \hat{f}_{\ell-1}+\frac{\ell+1}{2 \ell+3} \hat{f}_{\ell+1}\right) \\
+\frac{e E}{m}\left(\frac{\ell}{2 \ell-1} \frac{d \hat{f}_{\ell-1}}{d v}+\frac{\ell+1}{2 \ell+3} \frac{d \hat{f}_{\ell+1}}{d v}\right) \\
+\frac{e E}{m} \frac{1}{v}\left(\frac{\ell(\ell-1)}{2 \ell-1} \hat{f}_{\ell-1}-\frac{(\ell+1)(\ell+2)}{2 \ell+3} \hat{f}_{\ell+1}\right) \\
+J_{\ell} \hat{f}_{\ell}=0 \quad(\ell=0,1,2 \ldots) .
\end{aligned}
$$

This set of equations is solved by using the Galerkin method for six-term approximation. The computational procedure is the same as that in Date et al. ${ }^{14}$ [there are editorial errors in Eqs. (8) and (9) of the paper; however, the results are valid], and the six-term approximation applied to the present analysis has been validated by Yachi et al. ${ }^{16}$ The electron collision cross sections for methane were taken from Ohmori et al. ${ }^{19}$

\section{RESULTS AND DISCUSSION}

Figures 2(a)-2(d) show the collector current by the second shutter $\left(\mathrm{S}_{2}\right)$ as a function of drift distance at $1 \mathrm{~mm}$ intervals for $E / N=200,300,400$, and $500 \mathrm{Td}(1 \mathrm{Td}$ $=10^{-17} \mathrm{~V} \mathrm{~cm}^{2}$ ), respectively. These curves represent the spatial profiles of the evolution of electron swarms at every given moment ( $T_{m}$ in the figures). In relatively low $E / N$ values such as $200 \mathrm{Td}$, the drift distance is large enough to obtain the entire profile, with respect to space, of an isolated swarm. Contrary, in higher $E / N$ s, it was difficult to observe both-side tails of the distribution as shown in Figs. 2(b)-2(d) since the swarm moves with high speed drift and diffusion during a few nanoseconds of time intervals. In order to deduce the center-of-mass of the distribution at every moment even from such a set of short data, the smoothing spline technique (e.g., de Boor ${ }^{20}$ ) was employed to compensate for lack of data in between and tail parts. We confirmed that the data points below $5 \%$ of the peak value scarcely contribute to the determination of the center-of-mass of the distribution. Then, we plotted the center-of-mass as a function of corresponding time and deduced the drift velocity $\left(W_{r}\right)$ as the slope of approximate line for the plotted points by means of the least-squares method. The total error introduced by a series of the procedure was roughly estimated to be $4 \%$. In addition to $W_{r}$, we also deduced the mean-arrival-time drift velocity $\left(W_{m}\right)$ from the arrival-time distribution of electrons at fixed positions in the manner same as Hasegawa et al. ${ }^{9}$

The drift velocity obtained in the present experiment is shown in Table II and Fig. 3 with the computational results. The data by other investigators are also plotted in Fig. 3 for comparison. The experimental studies in methane have been reported by investigators such as Al-Amin et al., ${ }^{21}$ Haddad, $^{23}$ 
TABLE II. Results of the experimental and theoretical analyses.

\begin{tabular}{|c|c|c|c|c|c|c|c|c|c|c|c|}
\hline & \multicolumn{11}{|c|}{ Field strength $E / N(\mathrm{Td})$} \\
\hline & 100 & 200 & 250 & 282.3 & 300 & 350 & 400 & 424.2 & 450 & 500 & 565.6 \\
\hline \multicolumn{12}{|c|}{$W_{r}\left(\times 10^{7} \mathrm{~cm} / \mathrm{s}\right)$} \\
\hline Experiment & $\cdots$ & 1.41 & 1.80 & $\cdots$ & 2.20 & 2.69 & 3.17 & $\cdots$ & 3.63 & 4.05 & $\cdots$ \\
\hline Boltzmann eq. analysis & 0.771 & 1.45 & $\cdots$ & 2.07 & 2.20 & $\cdots$ & 3.02 & 3.23 & $\cdots$ & 3.90 & 4.46 \\
\hline \multicolumn{12}{|c|}{$W_{m}\left(\times 10^{7} \mathrm{~m} / \mathrm{s}\right)$} \\
\hline Experiment & $\cdots$ & 1.38 & 1.81 & $\cdots$ & 2.05 & 2.44 & 2.77 & $\cdots$ & 3.12 & 3.42 & $\cdots$ \\
\hline Boltzmann eq. analysis & 0.769 & 1.39 & $\cdots$ & 1.90 & 2.01 & $\cdots$ & 2.62 & 2.76 & $\cdots$ & 3.21 & 3.59 \\
\hline
\end{tabular}

Schmidt, ${ }^{24,25}$ Hunter et al., ${ }^{22}$ and Yoshida et al. ${ }^{26} \operatorname{Haddad}^{23}$ and Schmidt, ${ }^{24,25}$ however, focused on the study in low $E / N$ below $10 \mathrm{Td}$. In Fig. 3, the drift velocities taken from AlAmin $e t a l .^{21}$ and Hunter $e t a l .{ }^{22}$ are denoted by $W$ because it is unclear whether their velocities are equivalent to $W_{r}$ or $W_{m}$. As is shown in Fig. 3, the present experimental drift velocities are in fairly good agreement with other corresponding results. Slight differences are recognized between the experimental and computational results for $W_{r}$ and $W_{m}$, respectively, at high $E / N \mathrm{~s}$. As to these differences, other than the error in the experiment, we cannot rule out the possibility that the computational results have systematic errors arising from the incorrectness of electron collision cross section data for methane gas in the code. However, the difference between $W_{r}$ and $W_{m}$ is clearly exhibited and follows the same trend for both the experimental and computational values. The difference is up to about $16 \%$ at $E / N=500 \mathrm{Td}$. The relation between $W_{r}$ and $W_{m}$ is theoretically given ${ }^{6}$ by

$$
W_{m}=W_{r}-2 \alpha^{(0)} \omega^{(2)}+3 \alpha^{(0)^{2}} \omega^{(3)}-+\ldots,
$$

where the notations are the same as in Sec. III. The difference shown in the experiment ought to account the sum of second and higher terms in the right hand side of this equation.

Figure 4 shows the relation of $i k-\omega$ that was obtained from the Fourier transformed Boltzmann equation analysis.

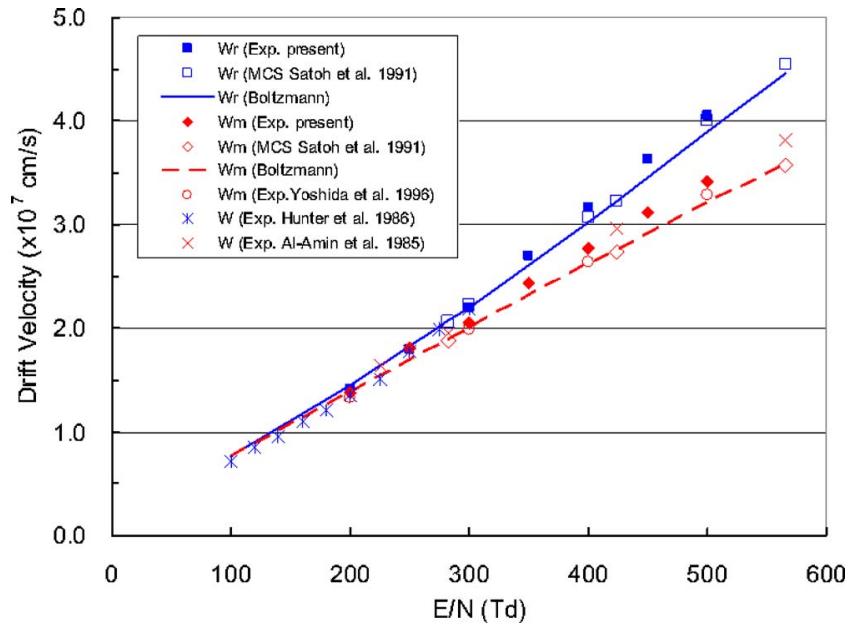

FIG. 3. (Color online) Electron drift velocities as a function of $E / N$ in methane in comparison with experimental measurements and theoretical analyses. In the legend, the drift velocities by Al-Amin et al. (Ref. 21) and Hunter et al. (Ref. 22) are denoted by $W$, and Exp. stands for experimental and MCS for Monte Carlo simulation.
By the curve fitting of polynomial Eqs. (9) and (11) to this relation, the velocities denoted with "Boltzmann" in Table II and Fig. 3 were obtained. Equations (9) and (11) tell us that the slopes of tangential lines at intersecting points on the vertical and horizontal axes give drift velocities $W_{r}$ and $W_{m}$, respectively. Note that the curves in Fig. 4 are not linear, and therefore these velocities are not identical. The difference between the definitions for $W_{r}$ and $W_{m}$ may be emphasized by following simple formulae:

$$
\begin{gathered}
W_{r}=\frac{\left\langle z_{i}\right\rangle-\left\langle z_{j}\right\rangle}{t_{i}-t_{j}}, \\
W_{m}=\frac{z_{i}-z_{j}}{\left\langle t_{i}\right\rangle-\left\langle t_{j}\right\rangle} .
\end{gathered}
$$

Here, the subscripts $i$ and $j$ represent different two points in time or space ( $z$-axis) and \langle\rangle denotes the mean value observed at a corresponding time or spatial location. Equations (16a) and (16b) imply that $W_{r}$ is deduced from the difference of mean-location of electrons at fixed two moments, while $W_{m}$ is from the difference of mean-time of electrons arriving at fixed two locations. The quantitative difference of these velocities is attributable to the effect of ionization (and/or attachment) events. ${ }^{6}$ In an isolated swarm, the electrons in forward part of the swarm have generally higher energies than those in rear part because they gain more potential energies from the electric field. Accordingly, the ionization oc-

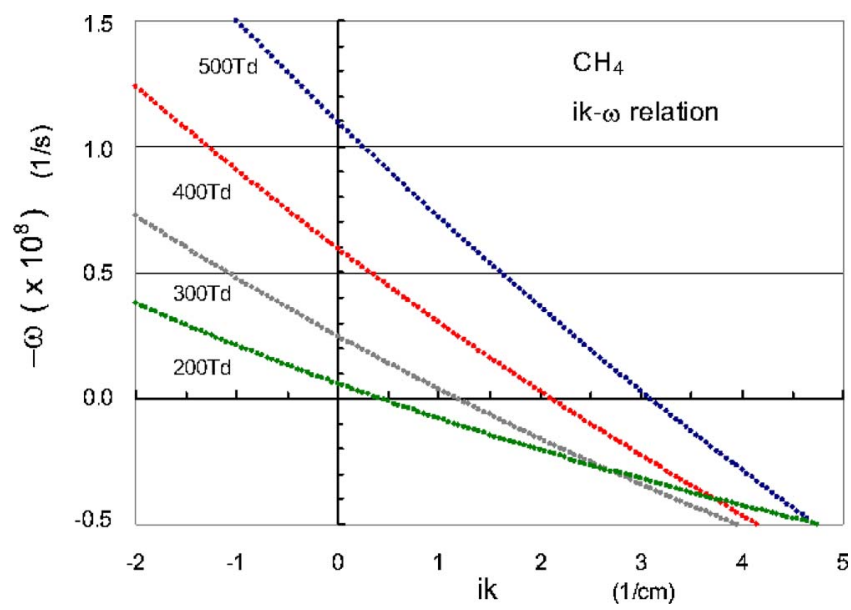

FIG. 4. (Color online) A dispersion relation, $i k$ vs $\omega$. Note that the curves are not linear, and therefore the slopes of tangent lines at the intercepts on the vertical and horizontal axes are not identical, which represent the difference between $W_{r}$ and $W_{m}$. 
curs with different frequencies in forward and rear locations, affecting the spatial distribution of electrons with newly born electrons. As a resultant effect of the production (and/or annihilation) processes of electrons along with drift and diffusion motions, interchangeability of time and space in observing the spatiotemporal evolution of the swarm comes to be impaired. We believe this is an important aspect of the particle ensemble behavior when the hydrodynamic regime includes particle production (and/or annihilation) processes. It should be noted that the difference in the drift velocities does not arise under conservation conditions of electron number, i.e., when the ionization and/or attachment processes are negligible. In fact, the difference was not recognized in low $E / N$ region below $100 \mathrm{Td}$ for methane gas.

\section{CONCLUSION}

In this study, we have performed the experiment to observe the spatial evolution of electron swarms at every moment, namely, "snap shots," using a double-shutter technique and conducted the theoretical analysis to solve a Fourier transformed Boltzmann equation. The experiment exemplifies the behavior of electron ensemble associated with drift, diffusion, and ionization (and/or attachment) phenomena as a function of time, in which we intend to make a direct comparison with the conventional TOF theory. The center-ofmass drift velocity $\left(W_{r}\right)$ defined as a typical TOF parameter was determined for methane gas and compared with the mean-arrival-time drift velocity $\left(W_{m}\right)$ based on the opposite observation principle between time and space. The result showed that the difference between them is significant at $E / N$ s above $200 \mathrm{Td}$, which is caused by the ionization process with the evolution of asymmetric profiles at high $E / N$, providing a direct basis for understanding the divergence of drift velocities depending on the observation methods.

\section{ACKNOWLEDGMENTS}

The authors are most grateful to Professor H. Tagashira and Professor K. Kondo for helpful discussions and useful suggestions. This work was financially supported by Grantsin-Aid for Scientific Research of Japan.

${ }^{1}$ K. Kumar, H. R. Skullerud, and R. E. Robson, Aust. J. Phys. 33, 343 (1980).

${ }^{2}$ J. H. Parker Jr. and J. J. Lowke, Phys. Rev. 181, 290 (1969).

${ }^{3}$ W. R. L. Thomas, J. Phys. B 2, 551 (1969).

${ }^{4}$ H. R. Skullerud, J. Phys. B 2, 696 (1969).

${ }^{5}$ H. Tagashira, Y. Sakai, and S. Sakamoto, J. Phys. D 10, 1051 (1977).

${ }^{6}$ K. Kondo and H. Tagashira, J. Phys. D 23, 1175 (1990).

${ }^{7}$ H. Date, K. Kondo, and H. Tagashira, J. Phys. D 23, 1384 (1990).

${ }^{8}$ H. Hasegawa, H. Date, M. Shimozuma, K. Yoshida, and H. Tagashira, J. Phys. D 29, 2664 (1996).

${ }^{9}$ H. Hasegawa, H. Date, and M. Shimozuma, J. Phys. D 40, 2495 (2007).

${ }^{10}$ B. Schmidt, Comments At. Mol. Phys. 28, 379 (1993).

${ }^{11}$ Y. Nakamura, J. Phys. D 20, 933 (1987).

${ }^{12}$ R. Hegerberg, M. T. Elford, and H. R. Skullerud, J. Phys. B 15, 797 (1982).

${ }^{13}$ J. Fletcher, Gaseous Electronics and Its Applications, edited by R. W. Crompton M. Hayashi, and D. E. Boyd (Kluwer, Tokyo, 1991).

${ }^{14}$ H. Date, K. Kondo, S. Yachi, and H. Tagashira, J. Phys. D 25, 1330 (1992).

${ }^{15}$ K. Kondo and H. Tagashira, J. Phys. D 26, 1948 (1993).

${ }^{16}$ S. Yachi, H. Date, K. Kitamori, and H. Tagashira, J. Phys. D 24, 573 (1991).

${ }^{17}$ K. Satoh, Y. Ohmori, Y. Sakai, and H. Tagashira, J. Phys. D 24, 1354 (1991).

${ }^{18}$ R. E. Robson, Gaseous Electronics and Its Applications, edited by R. W. Crompton et al. (Kluwer, Tokyo, 1991).

${ }^{19}$ Y. Ohmori, K. Kitamori, M. Shimozuma, and H. Tagashira, J. Phys. D 19, 437 (1986).

${ }^{20}$ C. de Boor, A Practical Guide to Spline (Springer-Verlag, New York, 1978).

${ }^{21}$ S. A. J. Al-Amin, H. N. Kücükarpaci, and J. Lucas, J. Phys. D 18, 1781 (1985).

${ }^{22}$ S. R. Hunter, J. G. Carter, and L. G. Christophorou, J. Appl. Phys. 60, 24 (1986).

${ }^{23}$ G. N. Haddad, Aust. J. Phys. 38, 677 (1985)

${ }^{24}$ B. Schmidt, Nucl. Instrum. Methods Phys. Res. A 252, 579 (1986).

${ }^{25}$ B. Schmidt, J. Phys. B 24, 4809 (1991).

${ }^{26}$ K. Yoshida, T. Ohshima, Y. Ohmori, H. Ohuchi, and H. Tagashira, J. Phys. D 29, 1209 (1996). 